\title{
CONOCIMIENTOS Y PRÁCTICAS SOBRE LA HIGIENE DE MANOS EN TRABAJADORES SANITARIOS. ARMENIA 2008- 2009
}

\section{KNOWLEDGE AND PRACTICES OF HAND HYGIENE IN HEALTH WORKERS. ARMENIA 2008 - 2009}

\author{
Ángela Liliana Londoño Franco ${ }^{1}$, Marta Lucia Murillas Bedoya ${ }^{1}$ \\ ${ }^{1}$ Facultad de Ciencias de la Salud, Universidad del Quindío.
}

Recibido: Agosto 9 de 2010

Aceptado: Noviembre 11 de 2010

Correspondencia: Programa de Medicina, Universidad del Quindío, Av. Bolivar calle 12 norte Armenia Quindio. Correo electrónico: angelalilianal@uniquindio.edu.co

\section{RESUMEN}

Objetivo: describir conocimientos y prácticas relacionados con la higiene de manos en trabajadores sanitarios y con los resultados realizar sugerencias de intervención. Materiales y métodos: 99 personas entre médicos y enfermeras aceptaron participar y firmaron consentimiento informado; se realizó una encuesta de conocimientos y prácticas y se evaluó la técnica de higiene de manos de acuerdo a las directrices de la Organización Mundial de la Salud. Resultados: Se encontró una práctica adecuada en un $27.5 \%$, aceptable $38.5 \%$ y mala 34.1\%. Se relacionaron significativamente con la realización adecuada de la técnica de higiene de manos la experiencia laboral y el tipo de trabajador sanitario. La mayor frecuencia de la práctica adecuada se encontró entre quienes llevaban menos de 6 años (60\%) de actividad laboral mientras que fue más baja entre aquellos que llevaban 21 y más años trabajando (11.1\%). Los participantes conocían la importancia de la higiene de manos y las ventajas del uso de soluciones alcohólicas. El conocimiento de las indicaciones en general fue bueno y varió entre 70 y $98 \%$. Conclusiones: Es necesario buscar estrategias para incentivar la práctica de higiene de manos y la técnica adecuada de acuerdo a los lineamientos del Reto de la Alianza Mundial en pro de la seguridad del pacientes "Una atención limpia es una atención segura".

Palabras clave: higiene de manos, seguridad del paciente, conocimientos sobre higiene de manos, lavado de manos, higiene de manos con soluciones alcohólicas

\begin{abstract}
Objetive: describe knowledge and practices related to hand hygiene in health care workers and make suggestions for intervention with the results. Materials and methods: 99 people including physicians and nurses agreed to participate and signed informed consent; we conducted a survey of knowledge and practices and evaluated the technique of hand hygiene according to the guidelines of the World Health Organization. Results: Good practice was found in $27.5 \%$ of the people, fair in $38.5 \%$ and poor in $34.1 \%$. Work experience and the type of health worker were found to be significantly related to the proper or improper performance of the technique of hand hygiene. The increased frequency of good practice was found among those who had been workingvfor less than 6 years (60\%) while it was lower among those who had beeen working for 21 or more years (11.1\%). The participants knew the importance of hand hygiene and the benefits of using alcohol solutions. Knowledge of the information was generally good and ranged between 70 and 98\%. Conclusions: It's necessary to find strategies to encourage the practice of hand hygiene and proper technique according to the guidelines of the Challenge of World Alliance of patient safety "Clean Care is Safer Care"
\end{abstract}

Keywords: hand hygiene, patient safety, knowledge about hand hygiene, hand washing, hand hygiene with alcohol solutions 


\section{INTRODUCCIÓN}

Las infecciones relacionadas con la atención sanitaria (IRAS) son un evento adverso y afectan cada año a cientos de millones de pacientes en todo el mundo; a su vez pueden ocasionar afecciones más graves, hospitalizaciones prolongadas y discapacidades de larga duración; por su propia naturaleza, las IRAS tienen una etiología multifacética, relacionada con los sistemas y procesos de prestación de atención y limitaciones políticas y económicas de los sistemas de salud en los países, así como con el comportamiento humano condicionado por la educación, sin embargo, la mayoría de ellas pueden prevenirse $(1,2)$.

El conocimiento de los resultados en materia de prevención de IRAS a través de las prácticas adecuadas de la higiene de las manos, ha llevado a conocer la eficacia de nuevos enfoques; el Reto Mundial por la Seguridad del Paciente 2005- 2006: "Una atención limpia es una atención más segura» está centrando parte de su atención en mejorar las normas y prácticas de higiene de las manos en la atención sanitaria y en ayudar a aplicar las intervenciones eficaces $(1,3)$; aunque se trata de una acción sencilla, su incumplimiento entre los dispensadores de atención sanitaria representa un problema en todo el mundo; en diversos estudios los hallazgos revelan los factores que influyen en el cumplimiento de las recomendaciones para la higiene de las manos (1); entre ellos se encuentran trabajar en unidades de cuidado intensivo o turnos durante la semana (comparado con el fin de semana), la utilización de medidas como bata y guantes que disminuye la adherencia a esta práctica, falta de personal o demasiado trabajo; los hallazgos también indican que existen diferencias entre los trabajadores sanitarios (TS), la adherencia es mayor entre las enfermeras que en las auxiliares de enfermería y médicos(1).

Los trabajadores sanitarios (TS) también encuentran dificultades en el cumplimiento de las indicaciones para la higiene de manos durante la atención porque los productos para el lavado de las manos causan irritación y sequedad, o no hay disponibilidad de jabón, toallas desechables o acceso fácil a los lavamanos; algunos tiene la idea de que la higiene de las manos interfiere la relación entre el profesional sanitario y el paciente, piensan que tienen bajo riesgo de contraer infecciones contagiadas por los pacientes y que los guantes reemplazan la higiene de manos; aunado a éstos factores se informa que no existen protocolos en la mayoría de las instituciones ni medidas para vigilar y controlar el cumplimiento de las indicaciones de dicha práctica $(1,2)$. El presente estudio se realizó con el objetivo de describir conocimientos y prácticas relacionados con la higiene de manos en TS que laboraban en servicios de hospitalización, quirófanos y urgencias. Se pretendió con los resultados realizar sugerencias de mejora realizando recomendaciones específicas a las instituciones de atención de pacientes.

\section{MATERIALES Y MÉTODOS}

Se realizó un estudio descriptivo prospectivo de los conocimientos y prácticas relacionadas con el lavado de manos con agua y jabón y la higiene con soluciones alcohólicas. La población de referencia fueron los TS de una institución hospitalaria y se incluyeron médicos, enfermeras y auxiliares de enfermería. Se invitaron a participar todas las personas de los diferentes servicios de hospitalización, urgencias y quirófanos y por medio de consentimiento informado, accedieron a participar 99 personas; a pesar de ser voluntaria la participación, se conservó la proporción de TS que laboraban por lo cual el $72,7 \%$ de los participantes fueron auxiliares de enfermería, médicos $18,3 \%$ (de ellos $7,1 \%$ médicos generales, $7,1 \%$ médicos internos y 4,0 médicos especialistas) y enfermeras jefes $9,1 \%$. El tamaño de la muestra seleccionada fue por conveniencia

Se aclara que muchos de los trabajadores sanitarios encuestados laboraban en varias instituciones; por tanto las respuestas no se refieren a las condiciones del hospital donde se realizaron las encuestas y pueden reflejar las condiciones generales en que se desarrolla la práctica de HM por parte de los participantes.

Técnicas generales y procedimientos para la recolección de la información: Se diseñó un instrumento para recolección con las variables de estudio, la información fue recolectada por una enfermera investigadora con la colaboración de un auxiliar de investigación y para garantizar la veracidad de la información, se explicaba a varios trabajadores sanitarios el contenido de la encuesta y después se entregaba para ser diligenciada sin consignar datos de identificación en la misma. Se realizó además una valoración de la práctica de higiene 
de manos de acuerdo a la técnica sugerida por la Organización Mundial de la salud (OMS) calificando con 1 o 0 si se incluía o no en el procedimiento de HM utilizando los siguientes ítems: palmas, espacios interdigitales anteriores, dorso manos, espacios interdigitales posteriores, pulgares, dorso dedos y punta dedos; con un puntaje máximo de 7 se definió como técnica adecuada si se obtenía un puntaje de 6 o 7 , aceptable 4 a 5 y mala de 0 a 3 .

Procesamiento y análisis de la información: el procesamiento y análisis de los datos se realizó en el programa SPSS versión 14. Se realizó análisis descriptivo con distribuciones de frecuencia para las variables cualitativas y medidas de tendencia central y posición para las cuantitativas. Se buscaron diferencias significativas con un nivel de confianza de un $95 \%$, se realizó test de chi cuadrado para buscar diferencias entre las variables cualitativas.

\section{RESULTADOS}

Se incluyeron 99 TS; personal de enfermería $81,8 \%$ y médicos $18,3 \%$. La media de años de experiencia en el sector salud de los participantes fue de 16.7 años (IC95\% 14.5-18.9), con un máximo de 30.3 años; el 10\% llevaba menos de un año de práctica.

Se preguntó acerca de la importancia de la higiene de manos (HM) en la atención de los pacientes, el 93.9\% justificaron la práctica en la prevención de infecciones, de ellos el $39.8 \%$ dio igual importancia a la prevención en pacientes y personal de salud, el $24.7 \%$ solo a la prevención de infecciones en pacientes y los demás se refirieron en general a la prevención de infecciones (prevención de infecciones $26.9 \%$, las manos son foco de infecciones 6.5\%).

Un 49\% de los encuestados afirmó conocer las directrices de la OMS sobre la higiene de manos y el lavado con agua y jabón. Se realizó una práctica de lavado de manos con agua y jabón encontrando que la duración del lavado de manos en promedio fue de 52 segundos (IC95\% 45- 59) variando entre 10 y 250 segundos, se presentaron varios valores extremos con duración mayor a un minuto y medio; sin embargo la mediana fue de 42 segundos. Se encontró una práctica ADECUADA en un $27.5 \%$, ACEPTABLE $38.5 \%$ y MALA $34.1 \%$. En primer lugar y con respecto a la práctica ADECUADA se encontraron los siguientes hallazgos: fue realizada por un $75 \%$ de los médicos siendo esta frecuencia significativamente mayor que entre las enfermeras con un $17.3 \%(p<0.000)$. Entre los médicos quienes realizaron con mayor frecuencia la práctica adecuada fueron los médicos internos con (6 de 12), le siguieron los especialistas 83 de 4) y por último los médicos generales ( 3 de 5 ). Entre las enfermeras, fue mayor entre las enfermeras jefes con $35.7 \%$ ( 3 de 8 ) y 14.9\% (10 de 67) entre las auxiliares de enfermería (Tabla 1). Al analizar la frecuencia con respecto a la antigüedad en el ejercicio, se encontró que era significativamente mayor entre las personas con menos de 5 años de experiencia (60\%), entre los 6 y 20 años disminuyó a $30.8 \%$ y después de los 20 años fue de $11.1 \%(p=0.04)$. Figura 1.

TABLA 1. Puntaje práctica de la técnica de higiene de manos

\begin{tabular}{lcccccc}
\hline \multicolumn{1}{c}{ TIPO DE TS } & \multicolumn{2}{c}{ ADECUADA 6-7 } & \multicolumn{2}{c}{ ACEPTABLE 4-5 } & \multicolumn{2}{c}{ MALA 0-3 } \\
\hline MD interno & № & $\%$ & № & $\%$ & № & $\%$ \\
MD general & 6 & 85.7 & 6 & 14.3 & 0 & 0.0 \\
MD especialista & 3 & 60 & 1 & 20 & 1 & 20 \\
TOTAL MEDIcOS & 3 & 75.0 & 1 & 25.0 & 0 & 0.0 \\
Enfermera profesional & $\mathbf{1 2}$ & $\mathbf{7 5 . 0}$ & $\mathbf{3}$ & $\mathbf{1 8 . 8}$ & $\mathbf{1}$ & $\mathbf{6 . 3}$ \\
Auxiliar enfermería & 3 & 37.5 & 4 & 50.0 & 1 & 12.5 \\
TOTAL ENFERMERAS & 10 & 14.9 & 28 & 41.9 & 29 & 43.3 \\
TOTAL & $\mathbf{1 3}$ & $\mathbf{1 7 . 3}$ & $\mathbf{3 2}$ & $\mathbf{4 2 . 7}$ & $\mathbf{3 0}$ & $\mathbf{4 0}$ \\
\hline
\end{tabular}




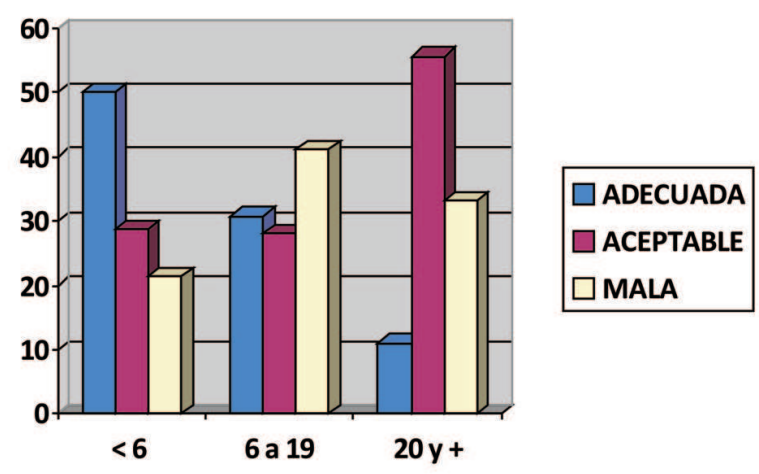

Figura 1. Técnica de higiene de manos según años de experiencia laboral

Se preguntó a los participantes que afirmaron conocer las soluciones con base en alcohol acerca de las ventajas y desventajas de las mismas; del total de respuestas un $42.3 \%$ de las respuestas tuvieron relación con la rapidez para el procedimiento (13 TS), la facilidad o lo práctico de las soluciones (11 TS), no necesita enjuague (1 TS), económicas (1 TS) y todas las respuestas (7 TS); en segundo lugar se encontraron las ventajas relacionadas con la capacidad antiséptica superior al lavado de manos (27 TS); la protección de las manos fue para los participantes una ventaja notoria pues la refirieron un $32.9 \%$ de los TS, entre las respuestas relacionadas estuvieron: no reseca y/o suaviza la piel (17 TS), no producen alergias y/o dermatitis (7 TS) y huelen bien(1 TS). Únicamente un 5,3\% refirieron desventajas entre las cuales se encontraron las siguientes respuestas: no tienen ninguna ventaja (2 TS), dejan las manos pegajosas (1 TS) y el acceso a ellas no es fácil (1 TS). Al preguntar si creían que las soluciones alcohólicas reemplazan la técnica habitual de lavado con agua y jabón, un $21.7 \%$ respondió que sí y un $78.7 \%$ respondió que no.
Se valoró el conocimiento sobre las indicaciones para la higiene de manos en la atención de los pacientes sugerido por la OMS encontrando que la mayoría conocían las indicaciones (Figura 2); cuando se preguntó si siempre se debía realizar lavado o higiene de manos, más del 95\% respondieron después del contacto con mucosas y con líquidos y excreciones; entre el 80 y $90 \%$ al tener contacto con piel no intacta y al quitarse los guantes, entre 70 y $80 \%$ después de examinar heridas, antes de manipular un dispositivo invasivo y después del contacto con pacientes; un $62 \%$ de los encuestados refirieron la práctica de $\mathrm{HM}$ al pasar de una zona contaminada a una limpia en la atención del mismo paciente. Predominó la respuesta a veces a la pregunta sobre realizar la $\mathrm{HM}$ antes del contacto con el paciente $55.6 \%$, después del contacto con objetos de la vecindad de los pacientes $53.5 \%$ y antes de colocarse los guantes un $52.5 \%$. En la respuesta "nunca se realiza $\mathrm{HM}^{\prime \prime}$ se encontró que un $29.3 \%$ escogieron esta respuesta cuando se trata de tener contacto con los objetos en la vecindad del paciente.

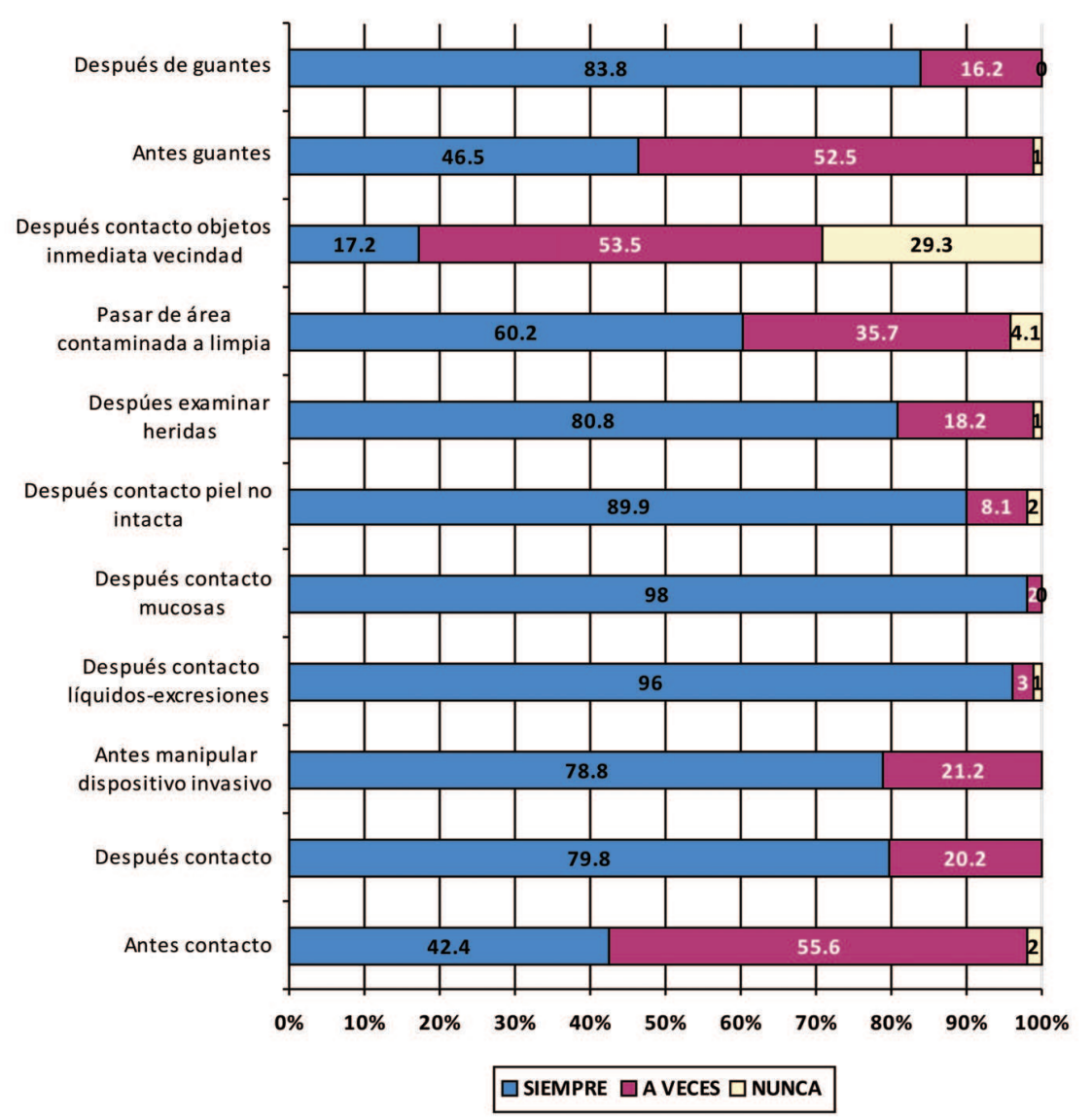

Figura 2. Cumplimiento de indicaciones para higiene de manos 


\section{DISCUSIÓN}

La transmisión cruzada se considera el principal mecanismo de diseminación de las infecciones hospitalarias, también conocidas como nosocomiales y más recientemente incluidas en un grupo de infecciones relacionadas con la atención sanitaria (IRAS); por tanto, la eliminación mecánica de los microorganismos de las manos y la utilización de sustancias con efecto bactericida realizando una técnica adecuada de lavado de manos o de higiene con soluciones alcohólicas, constituye la medida más importante de la prevención de dichas infecciones $(1,2)$.

La IRAS pueden afectar no solo a los pacientes sino a los TS, a los estudiantes de ciencias de la salud, a personal que labora en otras actividades en las instituciones de salud y a los visitantes, de ahí parte la gran importancia de prevenirlas $(4,5)$.

En el presente estudio, la mayoría de los TS consideraron la gran importancia de la higiene de manos para la prevención de infecciones, pero no todos consideraron el riesgo de infecciones ocupacionales y priorizaron la prevención de las mismas en los pacientes. Cerca de la mitad de las personas entrevistadas refirieron conocer un protocolo institucional relacionado con la higiene de las manos mientras que el resto lo desconocían. Tratándose del lavado rutinario, pero que determina la suspensión en la transmisión cruzada, llama la atención que menos de una tercera parte de los TS realizaran en forma adecuada la técnica de lavado de manos aunque la duración en todos los casos es adecuada y que la mayoría de TS que la hacen bien son los médicos encontrando una frecuencia muy baja de práctica adecuada sobre todo entre las auxiliares de enfermería.

Se observó un conocimiento adecuado acerca de la existencia, la forma de utilización, así como de las ventajas y desventajas de la utilización de soluciones alcohólicas, sin embargo se evidenció el desconocimiento de las indicaciones precisas de cuando se requiere el lavado con agua y jabón, y cuando pueden utilizarse las soluciones con base en alcohol.

El conocimiento de las indicaciones para higiene de manos durante la atención de pacientes es relativamente bueno pero llamó la atención el desconocimiento de la indicación de antes de tener contacto con el paciente, el contacto con objetos de la vecindad y antes de colocarse los guantes.

Aun conociendo la eficacia de la higiene de manos para prevenir la infección cruzada, se ha descrito que el cumplimiento de las indicaciones es pobre entre los TS $(6,7)$; incluso en lugares con condiciones ambientales óptimas, el cumplimiento se realiza entre el 50 a $60 \%$ la mayoría de veces (6); los hallazgos sugieren que factores del comportamiento como la actitud, influencias sociales pueden tener un papel en el cumplimiento (6). Existen estudios que difieren en la frecuencia de la práctica entre los TS; algunos refieren que el peor cumplimiento se ha encontrado entre los médicos (7), en otros los médicos realizan la práctica cuando está indicada entre un $57.6 \%$ y un $75 \%$ y las enfermeras $62 \%$ (8). Con respecto a los conocimientos sobre la $\mathrm{HM}$ refieren que han recibido capacitación solo un $56.5 \%$ (8).

De Wandel et all en 2010 han encontrado un cumplimiento total del $82 \%$, con $79.2 \%$ para contacto con superficies en la vecindad del paciente, $98.8 \%$ al tener contacto con sangre; otros estudios han encontrado una variabilidad en el cumplimiento de la HM de acuerdo a las oportunidades entre 27 y $66 \%$ $(6,7,8)$. El promedio de cumplimiento de las indicaciones se ha informado del $54 \%$; como factores independientes asociados se han encontrado la "autoeficacia" en la higiene de manos y la mala actitud con respecto al tiempo destinado al cumplimiento (6); entre los médicos se han descrito factores como la elevada carga de trabajo $(7,8)$, actividades asociadas con alto riesgo de transmisión cruzada y laborar en servicios de cirugía , anestesiología, urgencias y cuidados intensivos (7). Otro factor asociado con el incumplimiento es la cantidad insuficiente de solución alcohólica o su disponibilidad y falta de elementos para el lavado de manos tales como toallas desechables para los TS $(7,8)$.

Factores predictores para la adhesión a la práctica de HM por parte de los médicos se han determinado la conciencia de ser observado, promover buen ejemplo entre los colegas, la actitud positiva hacia la HM después del contacto con el paciente y el fácil acceso a las soluciones alcohólicas (7). 
El cumplimiento de la estrategia de HM representa un reto para los TS; cuando se realiza en todas las oportunidades puede representar hacerlo hasta 100 veces en un día (9).

La HM es la medida más importante para reducir la transmisión de microorganismos entre una persona y otra, y entre dos sitios distintos en un mismo paciente $(2,4,5)$. Múltiples estudios confirman la mayor eficacia de las soluciones alcohólicas (HSA) que contienen etanol al $80 \%$ o alcohol isopropílico al $75 \%$, actualmente se recomienda su utilización como alternativa al lavado de manos con jabón normal o con jabones antimicrobianos dentro de la práctica hospitalaria (1,10-14).

Las directrices de la OMS sobre higiene de manos están fundamentadas en el sistema CDC/HICPAC para la clasificación de las recomendaciones de acuerdo al tipo de estudios epidemiológicos que las soportan, en orden de importancia son categorías 1A, 1B, 1C y II (). En cuanto al lavado de manos con agua y jabón que se realiza con el fin de eliminar la suciedad, materia orgánica, flora habitual y transitoria y evitar la transmisión de estos microorganismos de persona a persona, indican que no puede reemplazarse con HSA y deben lavarse las manos con agua y jabón en las siguientes situaciones: cuando las manos estén visiblemente sucias o contaminadas con material proteináceo, visiblemente manchadas con sangre u otros líquidos corporales, cuando haya sospechas fundadas o pruebas de exposición a organismos con capacidad de esporular (IB) y después de ir al baño (II).(1).

Cuando se realice lavado con agua y jabón, se deben secar las manos completamente con una toalla desechable y utilizar la toalla para cerrar el grifo (IB), además se recomienda no emplear agua caliente porque aumenta el riesgo de dermatitis (IB). Cuando no se disponga de jabón antimicrobiano pueden emplearse jabones simples líquidos, en pastilla (las pastillas de jabón deben ser pequeñas y colocarse sobre rejillas que faciliten el drenaje), en hojas o en polvo. (II). (1).

En todas las demás situaciones clínicas, en las indicaciones se recomienda utilizar de preferencia una preparación alcohólica para la antisepsia sistemática de las manos (IA), o lavarse las manos con agua y jabón (IB): antes y después del contacto directo con pacientes (IB); después de quitarse los guantes
(IB); antes de manipular un dispositivo invasivo (se usen guantes o no) como parte de la asistencia al paciente (IB); después de entrar en contacto con líquidos o excreciones corporales, mucosas, piel no intacta o vendajes de heridas (IA); al atender al paciente, cuando se pase de un área del cuerpo contaminada a otra limpia (IB); después de entrar en contacto con objetos inanimados (incluso equipo médico) en la inmediata vecindad del paciente (IB); Lavarse las manos con agua y un jabón simple o antimicrobiano, o frotárselas con una preparación alcohólica antes de manipular medicamentos o preparar alimentos (IB) y no utilizar jabones antimicrobianos cuando ya se haya utilizado una preparación alcohólica para la fricción de las manos (II). Se indica para aplicarlas, usar una dosis de producto de aproximadamente un mililitro, extenderlo por toda la superficie de las manos y friccionarlas hasta que queden secas (IB). $(1,2,15)$.

El cambio de actitud hacia la práctica de higiene de manos no es únicamente de capacitación al personal de salud; la "teoría de la acción razonada" de Nutbeam y Harris 1999 dice que la educación sobre la amenaza de enfermedad induce en la gente cambios en los comportamientos de riesgo (16); sin embargo, un estudio realizado en Ghana por Beth Scott et all en 2007 recoge la evidencia que contradice dicha premisa donde se encuentra que muchas intervenciones educativas solas no han conducido en la disminución de la morbimortalidad y propone un modelo diferente donde encontraron que el comportamiento y la capacidad de cambio están determinadas por tres componentes: el hábito, factores de motivación, el contexto y el medio ambiente (16). Para promover el hábito es necesario proveer de los materiales necesarios para que la higiene de manos se convierta en rutina, además su fomento se debe hacer en las etapas en que las personas están más susceptibles a la conducta como podrían ser la época de formación técnica o universitaria, la llegada a una institución en período de prueba.

El estudio en Ghana identificó que la "repugnancia" constituyó una fuerte motivación para el comportamiento higiénico (16), por tanto, el ambiente hospitalario además de contar con los implementos necesarios, debería mostrar las consecuencias negativas de la no práctica de $\mathrm{HM}$ a través de afiches o mensajes que conduzcan a que las personas al cambio. 
Para lograr el cambio de actitud de los TS hacia unas buenas prácticas en lo referente a la higiene de manos, también se ha hablado de la "autoeficacia" que corresponde a la actitud positiva frente a una práctica que a las personas les produce sensación de éxito o gratificación (6). Entre las estrategias sugeridas para lograr cambios del comportamiento positivos dirigidos a la autoeficacia en el cumplimiento de la higiene de manos se refieren la educación, la motivación, la vigilancia del cumplimiento de las indicaciones y la retroalimentación entre los equipos multidisciplinarios de TS (6).

Así mismo con el fin de promover cambios en el comportamiento se habla recientemente del "marketing social", estrategia que incluye a los pacientes, los proveedores de atención en salud, los visitantes y la sociedad inmediata a todos ellos, utiliza intervenciones de mensajes, comunicación estratégica, cambios organizacionales y ambientales que influencien de manera positiva el cumplimiento de la HM; este enfoque apenas se está probando para mejorar dicha práctica $(7,16)$.

Los contenidos que debe incluir un programa educativo para los TS incluyen: información sobre el impacto en la morbimortalidad y los mecanismos de transmisión de las IRAS enfatizando en la contaminación cruzada, la evidencia disponible sobre la eficacia de la HM, las indicaciones y técnica adecuadas para el lavado con agua y jabón, para el uso de soluciones alcohólicas, los momentos en que se realiza HM dentro de la atención del paciente y los diferentes productos que pueden utilizarse para la HM (7); se debe recordar incluir todas las personas que laboren en las instituciones de salud sin importar el departamento o servicio al que pertenecen (4).

\section{CONCLUSIONES}

Existe conocimiento sobre el papel de las manos en la transmisión de las IRAS a pacientes más no sobre el riesgo de infecciones ocupacionales en los trabajadores sanitarios.

La mayoría de los participantes desconocen la existencia de protocolos institucionales para la higiene de manos.

Solo la tercera parte de los participantes realizaron bien la técnica de lavado con agua y jabón, la peor práctica se observó entre las auxiliares de enfermería y la mejor entre los médicos.
Existe conocimiento adecuado sobre los preparados en base de alcohol para antisepsia de las manos.

Existe desconocimiento sobre todos los "momentos" y las directrices para lavado con agua y jabón o con solución alcohólica.

\section{RECOMENDACIONES}

En las instituciones de atención sanitaria debe existir disponibilidad de elementos para el lavado de manos con jabón tales como lavamanos en sitios estratégicos, papel desechable y jabón suficiente; las soluciones con base en alcohol deben estar cerca a las camas de los pacientes.

Es necesario que desde la formación técnica o universitaria de los trabajadores sanitarios, la higiene de las manos constituya un pilar que fomente una actitud positiva ante esta medida.

Realizar educación continua en los centros de atención a pacientes que incluya los momentos para realizar la higiene de manos, las indicaciones específicas para el lavado con agua y jabón o con preparados con base de alcohol y las ventajas que tiene la prevención de infecciones en los pacientes y las infecciones ocupacionales en los trabajadores.

Elaboración de protocolos sencillos y concretos para que estén disponibles en todo momento.

Afiches en sitios visibles con las indicaciones y los momentos para la higiene de manos.

Personas encargadas de vigilar que se cumplan los protocolos y que se haga retroalimentación.

Involucrar los pacientes y visitantes motivándolos a mantener las manos limpias mientras dura el ingreso y continuar al alta. 


\section{BIBLIOGRAFÍA}

1. Organización Mundial de la salud. Alianza mundial para la seguridad del paciente. Directrices de la OMS sobre higiene de las manos en la atención sanitaria (borrador avanzado): Resumen unas manos limpias son manos más seguras. Ginebra 2005. Citado en: abril 2007. Disponible en: www.who.int/patientsafety

2. Organización Mundial de la salud. A Guide to the Implementation of the WHO Multimodal Hand Hygiene Improvement Strategy. Ginebra 2009. Citado en: abril 2010. Disponible en: www.who.int/patientsafety

3. Organización Mundial de la salud . Reto Mundial en pro de la seguridad del paciente 2005- 2006. Ginebra 2005. Citado en: abril 2010. Disponible en: www.who.int/patientsafety

4. OPS. Guía para la prevención y el control de las infecciones en servicios de salud, dirigida a estudiantes de las carreras de ciencias de la salud. Organización Panamericana de la Salud. 2007. Citado en: mayo 2010. Disponible: http://www.ops.org.bo/textocompleto/nis28679.pdf

5. OMS. Prevención de las infecciones nosocomiales. Guía práctica. Organización mundial de la salud 2003.

6. - De Wandel David, Maes Lea, Labeau Sonia, Vereecken Carine, Blot Stijn. Behavioral Determinants of Hand Hygiene Compliance in Intensive Care Units. Am J Crit Care. 2010; 19: 230-239. Citado: septiembre de 2010. Disponible: http://www.ajcconline.org

7. Johnston B. Lynn, Bryce Elizabeth. Hospital infection control strategies for vancomycin resistant Enterococcus, methicillin-resistant Staphylococcus aureus and Clostridium difficile. CMAJ MARCH 17, 2009 180(6) p 627631 Citado: septiembre de 2010. Disponible http://www.cmaj.ca/cgi/reprint/180/6/627

8. Anaya-Flores Verónica Edith, Ortiz-López Santa, Hernández-Zárate Victoria Elvia, García-Hernández Angélica, Jiménez-Bravo Ma. Leonor, Ángeles-Garay Ulises. Prevalencia de lavado de manos y factores asociados al incumplimiento. Estudio de sombra. Rev Enferm Inst Mex Seguro Soc 2007; 15 (3): 141-146. Citado: septiembre de 2010. Disponible: http://www.imss.gob.mx/NR/rdonlyres/7D93865D-4E6D-4C35-BFCACA15EBDD3B9D/0/RevEnf3052007.pdf

9. Kilbride Howard W., Wirtschafter David D., Powers Richard J, Sheehan Michael B. Nosocomial Infections Implementation of Evidence-Based Potentially Better Practices to Decrease. Pediatrics 2003;111;e519-e533. Citado: septiembre de 2010. Disponible: http://www.pediatrics.org/cgi/content/full/111/4/SE1/e519

10. Daniel H, Sh, de Mello AC, Jordao SJ, Sarto S V, Cassiolato V, Botelho GV, Cardoso C L. The effectiveness of alcohol gel and other hand-cleansing agents against important nosocomial pathogens. Brazilian journal of microbiology (2004) 35:33-39. Citado: septiembre de 2010. Disponible:

http://www.scielo.br/pdf/bjm/v35n1-2/arq05.pdf

11. Angeles G U, Molinar-R F, Anaya F V, López-G M. Efectividad de la aplicación de alcohol gel en la higiene de las manos de enfermeras y médicos. Rev enferm imss 2005; 13(1): 15-21 Citado: septiembre de 2010. Disponible: http://stesso.com.mx/gel/alcohol_gel.pdf

12. Yukiko Yamamoto RN, Kazuhiro Ugai, ; Yasuko Takahashi,. Efficiency of hand drying for removing bacteria from washed hands: comparison of paper towel drying with warm air drying. Infection control and hospital epidemiology vol. 26 no. 3 (316-320)march 2005 Citado: mayo de 2010.

Disponible:http://www.journals.uchicago.edu/doi/pdf/10.1086/502546 
13. Pellowe C M, Pratt R J, Loveday H P, Harper P, Robinson N, Jones S. The epic project. updating the evidencebase for national evidence-based guidelines for preventing healthcare-associated infections in NHS hospitals in England: a report with recommendations. British journal of infection control December 2004 vol. 5 no. 6 (10-16) Citado: mayo de 2010.

Disponible:http://www.epic.tvu.ac.uk/PDF\%20Files/epic\%201/epic\%20update.pdf

14. Boyce John M. Using alcohol for hand antisepsis: dispelling old myths. Infection control and hospital epidemiology july 2000. Editorial. vol. 21 no. 7 (438- 441). Citado: mayo de 2010. Disponible http://www.journals.uchicago.edu/doi/pdf/10.1086/501784

15. Health protection Scotlland, National services Scotland. Standard Infection Control Precautions (SICPs) Policies. Septiembre de 2009 p:1-20 Citado: mayo de 2010. Disponible:

http://www.documents.hps.scot.nhs.uk/hai/infection-control/sicp/handhygiene/mic-p-handhygiene-200909.pdf

16. Scott Beth, Curtis Val, Rabie Tamer, Garbrah- Aidoo Nana. Health in our hands, but not in our heads: understanding hygiene motivation in Ghana. Health Policy and Planning 2007;22:225-233 Citado: mayo de 2010. Disponible: http://heapol.oxfordjournals.org/content/22/4/225.full.pdf+html

17. López Serrato Manuel, Fernández Santiago Eloísa, Lebrero Ferreiro Rafael, Vázquez Garijo Pilar. Implementación de la Práctica Segura Higiene de Manos en Atención Primaria Subdirección de Coordinación de Salud Dirección General de Asistencia Sanitaria Servicio Andaluz de Salud. Abril 2008 . Citado: mayo de 2010. Disponible:

http://www.juntadeandalucia.es/agenciadecalidadsanitaria/observatorioseguridadpaciente/gestor/sites/Po rtalObservatorio/es/galerias/descargas/Implementacion_Practica_Segura_Higiene_Manos_AP_jun08.pdf 